\title{
Modelling of low energy ion sputtering from oxide surfaces
}

T Kubart ${ }^{1,2,3}$, T Nyberg $^{1}$, S Berg $^{1}$

${ }^{1}$ Solid State Electronics, The Ångström laboratory, Uppsala university, Box 534, 75121 Uppsala, Sweden

Tel.: +46 18471 7257; Fax: +46 18555 095;

${ }^{2}$ Department of Control Engineering, Faculty of Electrical Engineering, Czech Technical University in Prague, Technická 2, Prague 6, Czech Republic

\begin{abstract}
The main aim with this work is to present a way to estimate values of the surface binding energy for oxides. This is done by fitting results from the binary collisions approximation code Tridyn, with data from the reactive sputtering processing curves, as well as the elemental composition obtained from X-ray photoelectron spectroscopy (XPS). Oxide targets of $\mathrm{Al}, \mathrm{Ti}, \mathrm{V}, \mathrm{Nb}$, and Ta are studied The obtained surface binding energies are then used to predict the partial sputtering yields. Anomalously high sputtering yield is observed for $\mathrm{TiO}_{2}$ target. This is attributed to the high sputtering yield of Ti lower oxides. Such an effect is not observed for the other studied metals. XPS measurement of the oxide targets confirms the formation of suboxides during ion bombardment as well as an oxygen deficient surface in steady state. These effects are confirmed from the processing curves from the oxide targets showing an elevated sputtering rate in pure argon.
\end{abstract}

\section{PACS: $81.15 \mathrm{Cd}$}

Keywords: Sputtering of oxides, Surface binding energy, Reactive sputtering, Sputtering yields, Tridyn

\footnotetext{
${ }^{3}$ E-mail: Tomas.Kubart@angstrom.uu.se
} 


\section{Introduction}

Physical sputtering is employed in many applications, such as ion beam sputtering used for surface cleaning, depth profiling [1], or pattering and etching [2]. Magnetron sputtering is on of the most common industrial coating technique. The process of low energy sputtering is well understood and has been extensively studied for elemental targets. Both analytical theories and computer modelling have been employed [3].The picture gets more complex when sputtering from multi-component targets is considered, i.e. when partial sputtering yields and the surface composition evolution are of interest [4]. In linear cascade regime the ratio of partial yield is proportional to the component mass and indirectly proportional to the surface binding energy [5]. Several attempts have been carried out to find an expression for the surface binding energy [6,7]. The results were always limited to certain group of compounds or elements. Due to the limitations of analytical theories, computer modelling has been used for the description of sputtering events. The most common approach is based on binary collision approximation (BCA), as other more complex methods such as molecular dynamics require too much computer time in case of dynamic simulations [3]. However, the drawback of binary collision models is that the surface binding energy has to be defined as one of the input parameters.

Partial sputtering yields are of particular interest in sputter deposition processes, where they give information on the achievable deposition rates. In reactive sputtering, where a reactive gas is added to the conventional argon working atmosphere, the main issue is related to the hysteresis effect. The hysteresis effect limits the achievable compositions and decreases the deposition rate substantially. The hysteresis is normally very pronounced in deposition of oxides partly due to the high difference in metal and oxide sputtering yield [8]. It is frequently necessary to employ closed loop control of the reactive gas pressure to obtain high deposition rates and optimum film properties [9]. Hysteresis may be avoided by the use of an oxide target instead of pure metal. However, insulating target materials require RF sputtering with complicated and costly hardware. RF sputtering also generally achieves lower deposition rates. Moreover, for large area depositions, the setup gets very complex. Computer modelling is therefore an important tool in development of high deposition rate processes.

One of the still open questions concerns sputtering yields of oxides. From some experiments, sputtering yield of oxides have been reported close to the yield of corresponding metal [10]. This is, however, inconsistent with the observed reduction in deposition rate in reactive sputtering, when comparing metal and poisoned sputter erosion rate [11]. The authors of this study have previously 
presented a model explaining the mechanisms responsible for the high deposition rate in reactive sputtering of $\mathrm{TiO}_{2}$ [12]. In that article, the sputtering process using $\mathrm{TiO}_{\mathrm{x}}$ targets of various compositions was reported. Modelling of the process indicated that the high deposition rate is related to the presence of lower Ti oxides (suboxides) at the target surface. It was shown that the sputtering yield of Ti suboxides reaches very high values, comparable with metal targets. Other studies showed formation of suboxides on surfaces of other oxides such as Hf [13] and Ta [14] oxide. In this paper, we report on preferential sputtering of different oxide surfaces. The main motivation for the work is to extend the previous study to several other materials with relevance for thin film depositions.

\section{Experimental}

$\mathrm{X}$-ray photoelectron spectroscopy (XPS) is a surface sensitive method. Typical probing depth is few $\mathrm{nm}[15]$ and therefore it reveals information about the topmost surface. A PHI Quantum 2000 instrument with monochromatised $\mathrm{Al} \mathrm{K \alpha}$ radiation was used in this study. The typical resolution of measurements performed in this equipment is of the order $0.1 \mathrm{eV}$. Measurements of the $\mathrm{O} 1 \mathrm{~s}, \mathrm{Al} 2 \mathrm{p}$, Ta4f, Ti2p, V2p and Nb3d peaks were carried out on the surface prior to sputtering and after sputtering for different time periods. Sputtering was performed with a $2.0 \mathrm{keV} \mathrm{Ar}$ beam with an ion current of approximately $800 \mathrm{nA}$ onto an area of $4 \times 4 \mathrm{~mm}^{2}$, giving the current density of about $5 \mu \mathrm{A} / \mathrm{cm}^{2}$. Charging caused by the beam was compensated for by a neutraliser. The background pressure in the analytical chamber was of the order of $10^{-9}$ Torr.

The peaks were fitted by means of program XPSPEAK 4.1 assuming symmetrical peaks having $80 \%$ Lorentzian and 20\% Gaussian distribution. Moreover, the type of peak (s. p, d etc) and the spin-orbit splitting was given as input. Each peak was fitted with respect to peak position, area and FWHM. The peak area was calculated after subtracting a manually defined Shirley background and was used as a measure of the quantity of the oxide or metal belonging to the peak in question.

For each studied metal oxide, two series of magnetron sputtering experiments were carried out. First, a target made of pure metal was sputtered with variable oxygen mass flow. The experiment was then repeated under the same conditions with a target made of corresponding stoichiometric oxide. Sputtering was performed in a custom build sputtering system. The cylindrical sputtering system, with a chamber volume of 201 , was evacuated by a cryo pump. The system was equipped with a Lesker Torus 2" circular planar magnetron. In order to enable sputtering of oxide targets, a CESAR 1312 RF generator with Variomatch automatic matching network was used. 2" targets having a thickness of $3 \mathrm{~mm}$ were used. A quartz crystal microbalance (QCM) was used for the mass deposition rate 
measurements. Composition of the sputtering atmosphere was analyzed by a differentially pumped quadrupole mass spectrometer Spectra HPQ-2S. Partial pressures were calibrated by means of a capacitance manometer. The pumping system was throttled to a pumping speed of $30 \mathrm{l} / \mathrm{s}$ for oxygen. The base pressure achieved in this system prior to each deposition was below $3 \times 10^{-5} \mathrm{~Pa}$ at full pumping speed. This gave pressure of about $1.5 \times 10^{-4} \mathrm{~Pa}$ at the reduced pumping speed. Sputtering experiments were carried out in a sputter up configuration using an argon pressure of $0.4 \mathrm{~Pa}$ and a flow of $6.8 \mathrm{sccm}$. All parameters were kept constant, except the oxygen flow which was varied in order to obtain the whole hysteresis curve. Constant real power mode RF sputtering was performed at $60 \mathrm{~W}$. Mass deposition rate, oxygen partial pressure and RF self-bias was recorded as a function of oxygen flow.

\section{Modelling}

The surface composition of a multielement target generally changes during sputtering, leading to a formation of layer with a composition different from the bulk [4]. In order to simulate this, a dynamic description has to be used. In this work, the ion surface interaction has been simulated using the Tridyn code [16]. Tridyn is a BCA Monte-Carlo code frequently used for simulation of sputtering [17]. In the program, the target surface region is divided into depth slabs, initially equidistant. The changes in composition and thickness of each slab as a result of ion impact and subsequent collision cascades are followed. Concentration of each component may be limited by the stoichiometric limit. A local saturation model is employed in Tridyn, the atoms exceeding the local saturation limit are either directly reemited, or diffuse to the next slab towards the surface [18]. Argon incorporation is neglected in this simulation.

The most crucial parameter in a BCA calculation is the surface binding energy (SBE). It describes a planar surface potential which causes an energy loss and a refraction of sputtered atoms toward the surface [3]. Heat of sublimation is the most common choice for the surface binding energy of elemental targets [19]. The value is, however, usually not known for compound targets and an approximation based on the heat of atomization [20], in some cases corrected for electronegativity [7], is commonly used. Such an approach, however, is not realistic for oxides because the calculated values of compound sputtering yield [10] are generally substantially larger than the experimental values determined from deposition rate [12]. Therefore, we used the ratio of deposition rate in metal and compound mode to determine an appropriate value of SBE by fitting. 
In Tridyn, the SBE can be either constant, or dependent on the actual surface composition. In the later case, used for the metal in this study, the SBE of a component $\mathrm{A}$ (metal) in a binary compound $\mathrm{AB}$ is given by

$$
S B E_{A}=S B E_{A A} \cdot c_{A}+S B E_{A B} \cdot c_{B}
$$

where $\mathrm{c}_{\mathrm{A}}$ and $\mathrm{c}_{\mathrm{B}}$ is the surface concentration of the component $\mathrm{A}$ and $\mathrm{B}$, respectively. $S B E_{A A}$ corresponds to the $\mathrm{SBE}$ of pure metal, $S B E_{A B}$ is the $\mathrm{SBE}$ of oxidised metal in a stoichiometric compound. For the oxygen constant value of SBE was used, independent of surface composition. This is based on the assumption that majority of the oxygen present at the target is contained in oxide.

\section{Results and Discussion}

\section{XPS analysis}

XPS spectra from an oxide target surface are shown in Figure 1 for niobium oxide (a), and aluminium oxide target (b), respectively. Results of ion sputtering of the surface reveal a completely different picture. The $\mathrm{Nb}_{2} \mathrm{O}_{5}$ target shows a clear change with sputtering time and peaks corresponding to lower valence state appear. Further, there is a significant increase in the FWHM of the ion bombarded niobium oxide peaks as compared to FWHM from the virgin surface. This is due to the amorphization caused by the impinging ions. Alumina surface, on the other hand, remains unaffected. There are no lower $\mathrm{Al}$ oxides, hence the only possible surface states are $\mathrm{Al}$ and $\mathrm{Al}_{2} \mathrm{O}_{3}$. This behaviour is different from what is observed for all other metals investigated in this study. As seen in Figure 1b), the peak shape didn't change which indicates no change in the surface composition. This result is in agreement with other studies of ion bombarded alumina surfaces [6]. Absence of preferential sputtering is, however, in contradiction with the results of magnetron sputtering, shown later in Table II. All other studied oxides showed preferential sputtering of oxygen as a result of ion bombardment. Analogous to $\mathrm{Nb}$, Figure 1a), peaks corresponding to lower valences appear.

The spectra obtained for each target were fitted in order to determine the concentration of individual oxides (valence states). As an example, fitted results for $\mathrm{Nb}$ are shown in Figure 2 for oxide and metal target. Only the onset of sputtering during first 5 minutes is shown for the Nb target, the steady-state reported in Table I was reached after substantially longer sputtering time. Surface composition of each material, fitted from XPS, is summarized in Table I together with observed valences states. It should be noted, that in some cases it is not possible to distinguish between contribution of individual valence 
states (such as $\mathrm{Ti}^{2+}$ and $\mathrm{Ti}^{3+}$ ) and the choice is somewhat arbitrary. The result is sensitive to the position of the peaks and the obtained fractions in Figure 2 should be regarded as qualitative rather than quantitative. In addition, the amorphization caused by the impinging ions adds uncertainty to the results.

Ion beam etching experiments were carried out for metal targets as well. A layer of native oxide formed at the surface has a thickness of about $3 \mathrm{~nm}$ for machined Ti metal. Thickness of about $2 \mathrm{~nm}$ has been reported for native oxide on $\mathrm{Al}$ and Ta surfaces [21]. Considering the uncertainty, a thickness of $3 \mathrm{~nm}$ was assumed for all measured targets in simulations Metal targets showed stoichiometric oxides at the surface and after sputtering, peaks corresponding to the metal were dominating. In some cases, namely for $\mathrm{Al}$ and $\mathrm{Ti}$ target, the sputtering time to reach the steady-state is very long, in the order of hours. This is related to the low absolute value of alumina and titania sputtering rate.

\section{Sputtering experiments}

A typical result of the reactive sputtering experiment for metal and oxide targets is shown in Figure 3 for $\mathrm{Nb}$ and $\mathrm{Ti}$. For metal targets, there are two clearly separated working modes. In the so-called metal mode, the flow of oxygen is low enough and the target is kept metallic [8]. Therefore, the deposition rate is high and the partial pressure of oxygen low, as virtually all the oxygen is incorporated into the growing film. For higher oxygen flows, the target surface gets covered by oxide (poisoned) and the deposition rate drops as a result. This processing region is called compound mode. The abrupt transition from high rate metal mode to compound mode is accompanied by an equally abrupt change in the oxygen partial pressure, see Figure 3 a), and target voltage, not shown. The magnitude of the hysteresis depends on the target material (sputtering yields and reactivity) and the configuration of the sputtering system (target size, pumping speed, etc.). Ti is a very reactive metal with a high difference between metal and oxide sputtering rate. This results in a wide hysteresis. $\mathrm{Nb}$, on the other hand, shows very narrow loop, typical for less reactive systems, or materials with relatively high oxide sputtering rate.

The oxide targets showed no hysteresis as expected, for increasing oxygen flow both pressure and deposition rate changes continuously. However, we keep the same terminology also for oxide targets. Metal mode refers to the sputtering in pure Ar atmosphere while in compound mode sufficient flow of $\mathrm{O}_{2}$ is used. In compound mode, the deposition rate from both metal and related oxide target reached the same value, corresponding to sputtering from completely oxidised target. An increased content of $\mathrm{O}_{2}$ in the working gas may also change the deposition rate since oxygen has generally lower sputtering yield than Ar. The $\mathrm{O}_{2}$ flow is substantially lower than the $6.8 \mathrm{sccm}$ of $\mathrm{Ar}$ and thus this effect is 
considered to be negligible in our experiments. With the only exception of Ta, the compound mode was reached for flows well below $1 \mathrm{sccm}$.

Table II lists measured values of mass deposition rate in metal and compound mode as measured by QCM. A clear indication of the preferential sputtering is the difference of metal and compound deposition rate from an oxide target. Unaffected surface would have the same deposition rate independent of $\mathrm{O}_{2}$ flow rate, provided the $\mathrm{O}_{2}$ content in the sputtering atmosphere is low. The rates in pure argon are about 3 to 10 times higher than the compound rates, indication that the target surface changed as a result of ion bombardment. This is in agreement with XPS results, with the exception of Al. As seen in Table II, there is a pronounced drop in deposition rate for Al, Ti and V. In case of Ti, a relatively high rate difference between metal and compound rate from oxide target corresponds with previous observation [22]. $\mathrm{V}$, on the other hand, shows smaller difference, similar to $\mathrm{Al}$ despite the formation of lower oxide at the surface of $\mathrm{V}_{2} \mathrm{O}_{5}$ target.

\section{Tridyn simulations}

The SBE of oxidised metal $S B E_{A B}$ has been determined by fitting to experimental results, using following procedure:

The surface binding energy of oxidised metal was determined from the ratio of the deposition rate in metal and compound mode. The value of heat of sublimation was used for the SBE of pure metal. The $S B E_{A B}$ value used for the simulation was selected to reproduce the erosion rate measured in experiments. Simulations were carried out in Tridyn static mode, assuming operation in pure argon, and deep in compound mode, where the target surface remains completely oxidised. Argon energy of $200 \mathrm{eV}$ was used, corresponding to the mean value of target self bias. The bulk binding energy, defining the relocation energy threshold, was set to zero in all Tridyn calculation in agreement with the convention [17]. Oxygen concentration was not limited in this simulations, allowing for possible implantation of unreacted oxygen into the target [4]. The maximum observed concentration of oxygen was a few percent above the stoichiometric concentration.

XPS measurements, showing the steady-state surface composition of ion sputtered oxide target, were then used to determine the oxygen SBE. Fluence of $1.12 \times 10^{17} \mathrm{~cm}^{-2}$, corresponding to 60 minutes of sputtering, was used in order to reach a steady state. The dynamic calculations were performed at $2000 \mathrm{eV}$, the $\mathrm{Ar}^{+}$energy used in the experiment. The oxygen SBE was adjusted to reproduce the observed surface metal-oxygen ratio. 
Obtained SBE values are shown in Table III. The sputtering process of metals with high atomic mass is driven by the mass difference between the metal and oxygen. The SBE of $\mathrm{Ta}$ and $\mathrm{Nb}$ is not influenced by oxidation and constant SBE gives good results [23]. The energy transfer to the lighter component is enhanced, which results in higher oxygen sputtering yield. As a result, enhanced preferential sputtering of oxygen maintains the target in metal mode and is responsible for the absence of hysteresis in case of Ta and very narrow hysteresis for $\mathrm{Nb}$. For elements with lower atomic mass, the effect of binding is much more pronounced. There is a substantial change in the SBE for oxidised metals.

In order to verify the used approach, determined surface binding energies have been used to simulate the preferential sputtering of oxide targets during sputtering in Ar. Simulated results may then be compared with experimental values since these values were not used for fitting. The dynamical simulations were performed for $\mathrm{Ar}^{+}$bombardment at the energy of $200 \mathrm{eV}$, the mean value of the target self bias in the RF sputtering. The calculated partial sputtering yields of metal in steady-state are shown in Figure 4 together with measured values. All yields are normalized to the value of metal sputtering yields from stoichiometric oxide (targets operated in compound mode) and the relative yield is a measure of the extent of preferential sputtering. As seen from Figure 4, there is a reasonable agreement between simulations and experiments for all target materials, except Ti. For Ti, experimental sputtering yield exceeds simulation about three times. This indicates that a fundamentally different mechanism contributes to the sputtering. High sputtering yield of Ti suboxides has been shown to play an important role [12]. Tridyn doesn't take such an effect into account, the surface binding energy is simply linearly interpolated according to (1). V, although forming a lower oxide, did not show such a high erosion rate increase. The different behaviour of Ti and other metals has not been completely understood yet.

Using values from Table III, the evolution of surface composition has been simulated as a function of Ar energy. The results are shown in Figure 5 with oxygen surface concentration relative to the stoichiometric value. Preferential O sputtering is more pronounced at lower energies. Such effect has been reported for $\mathrm{Ta}_{2} \mathrm{O}_{5}[14]$.

In case of $\mathrm{Al}_{2} \mathrm{O}_{3}$ surface, there is a disagreement between XPS and sputter experiments. Increased erosion rate from such a target in magnetron sputtering indicates preferential sputtering of oxygen and surface enrichment by metal. Lack of bombardment induced mass transport has been suggested as the reason for no change observed by XPS [6,7]. In such case, only very thin altered surface is created which cannot be detected by XPS. Such behaviour has been recently reported for $\mathrm{ZnO}$ [24] where only 
one monolayer thick $\mathrm{Zn}$ enriched layer was after ion bombardment. Also the influence of residual gas in the XPS chamber may be pronounced for oxides with low sputtering rate.

\section{Conclusions}

The sputtering yields measured by ion beam sputtering of oxide targets may differ substantially from a value corresponding to an undisturbed oxide surface. Due to the preferential sputtering, the apparent partial sputtering yield of metal is much higher than from the unaffected surface. BCA based computer simulation codes give a good description of the sputtering event in the region of low energy sputtering. However, care has to be taken when choosing the values of surface binding energy for lighter metal oxides since the effect from binding is very strong. Fitted values, reported in this study, may be used in modelling of sputtering processes in order to describe the preferential sputtering and predict partial sputtering yields. It has been shown that there is a good agreement between simulated and measured target erosion rates for all studied metals with the exception of Ti. In case of Ti, there is a marked effect of lower oxides.

In case of $\mathrm{Al}_{2} \mathrm{O}_{3}$, there is a disagreement between XPS and sputter experiments. Possible explanation lies in the influence of residual gas, especially pronounced for oxides with low sputtering rate or formation of very thin and hence undetected layer at the surface.

Reported enhance preferential sputtering at lower energies may be important when ion beams are used for surface cleaning prior to surface analysis. The surface composition depends on the $\mathrm{Ar}^{+}$energy and the original composition is better preserved at higher energies.

\section{Acknowledgements}

This work was supported by the Swedish Foundation for Strategic Research (SSF) Strategic Research Center in Materials Science for Nanoscale Surface Engineering (MS ${ }^{2}$ E). Part of the project was also funded by Volkswagen Foundation. 


\section{Figure captions}

Figure 1: Evolution of XPS spectra of $\mathrm{Nb}_{2} \mathrm{O}_{5}$ (a) and $\mathrm{Al}_{2} \mathrm{O}_{3}$ (b) surface during $\mathrm{Ar}^{+}$bombardment. The arrow indicates increasing sputtering time. In figure (a), the binding energies for $\mathrm{Nb} \mathbf{3 d 5 / 2}$ peak in different oxides are indicated.

Figure 2: Surface evolution of $\mathrm{Nb}_{2} \mathrm{O}_{5}$ (a) and oxidised $\mathrm{Nb}$ (b) target surface under $2 \mathrm{keV} \mathrm{Ar}^{+}$ bombardment as measured by XPS.

Figure 3: Typical hysteresis curve in oxygen partial pressure (a) and mass deposition rate (b) normalized to the compound value. Results for $\mathrm{Ti}, \mathrm{TiO}_{2}, \mathrm{Nb}$, and $\mathrm{Nb}_{2} \mathrm{O}_{5}$ targets are shown.

Figure 4: Simulated and measured partial sputtering yields of metal from oxide targets sputtered in pure Ar. All yields are normalized to the values in compound mode.

Figure 5: Steady-state surface concentrations of oxygen relative to the stoichiometric concentrations as a function of $\mathrm{Ar}^{+}$energy. 


\section{Tables}

Table I: Target surface composition (fraction of oxygen at the target surface) and observed valence states in steady-state after $\mathrm{Ar}^{+}$bombardment.

\begin{tabular}{|c|c|c|c|c|c|c|c|c|c|c|}
\hline Target & $\mathrm{Al}$ & $\mathrm{Al}_{2} \mathrm{O}_{3}$ & $\mathrm{Ta}$ & $\mathrm{Ta}_{2} \mathrm{O}_{5}$ & $\mathrm{Ti}$ & $\mathrm{TiO}_{2}$ & V & $\mathrm{V}_{2} \mathrm{O}_{5}$ & $\mathrm{Nb}$ & $\mathrm{Nb}_{2} \mathrm{O}_{5}$ \\
\hline \multicolumn{11}{|l|}{ Oxygen } \\
\hline conc. [at.\%] & 25 & 60 & 15 & 54.7 & 14.5 & 59 & 0 & 63 & 9.2 & 67 \\
\hline Observed & & & Ta, & $\mathrm{Ta} \mathrm{Ta}^{2+}$, & $\mathrm{Ti}$ & $\mathrm{Ti}^{4+}$ & & $\mathrm{V}^{3+}$, & $\mathrm{Nb}$, & $\mathrm{Nb}^{2+}$ \\
\hline valence states & \multicolumn{2}{|c|}{$\mathrm{Al}, \mathrm{Al}^{3+} \mathrm{Al}^{3+}$} & $\mathrm{Ta}^{2+}$ & $\mathrm{Ta}^{5+}$ & $\mathrm{Ti}^{2+}$ & $\mathrm{Ti}^{3+}, \mathrm{Ti}^{2}$ & V & $\mathrm{V}^{5+}$ & $\mathrm{Nb}^{2+}$ & $\mathrm{Nb}^{4+}, \mathrm{Nb}^{5+}$ \\
\hline
\end{tabular}

Table II: Measured deposition rates in metal and compound mode for metal and oxide targets.

\begin{tabular}{|c|c|c|c|c|c|c|c|c|c|c|}
\hline Target & $\mathrm{Al}_{2} \mathrm{O}$ & ${ }_{3} \mathrm{Al}$ & $\mathrm{TiO}_{2}$ & $\mathrm{Ti}$ & $\mathrm{V}_{2} \mathrm{O}_{5}$ & V & $\mathrm{Nb}_{2} \mathrm{O}_{5}$ & $\mathrm{Nb}$ & $\mathrm{Ta}_{2} \mathrm{O}_{5}$ & $\mathrm{Ta}$ \\
\hline \multicolumn{11}{|l|}{ Mass dep. rate in metal } \\
\hline mode [a.u.] & 0.9 & 6.8 & 1.9 & 4.7 & 1.2 & 5.4 & 9.3 & 14.3 & 26.5 & 41.5 \\
\hline \multicolumn{11}{|l|}{ Mass dep. rate in } \\
\hline compound mode [a. u.] & 0.23 & 0.42 & 0.21 & 0.22 & 0.42 & 0.48 & 1.92 & 1.57 & 4.8 & 3.8 \\
\hline
\end{tabular}

Table III: Fitted surface binding energies and resulting steady-state surface composition (oxygen concentration) simulated by Tridyn.

\begin{tabular}{lllll}
\hline Element & $\begin{array}{l}\text { SBE(metal })_{\text {metal }} \\
{[\mathrm{eV}] \text { from }[19]}\end{array}$ & $\begin{array}{l}\text { SBE(metal) } \\
{[\mathrm{eV}]}\end{array}$ & $\begin{array}{l}\text { SBE(oxygen) } \\
{[\mathrm{eV}]}\end{array}$ & $\begin{array}{l}\text { Sim. surface } \\
\text { O conc. [at.\%] }\end{array}$ \\
\hline $\mathrm{Ti}$ & 4.86 & 10.0 & 7 & 53 \\
\hline $\mathrm{Al}$ & 3.36 & 12.0 & 9 & 56 \\
\hline $\mathrm{Ta}$ & 8.1 & 8.1 & 8 & 55 \\
\hline $\mathrm{Nb}$ & 7.6 & 7.6 & 9 & 61 \\
\hline $\mathrm{V}$ & 5.3 & 6.2 & 6 & 61 \\
\hline \hline
\end{tabular}




\section{Figures}
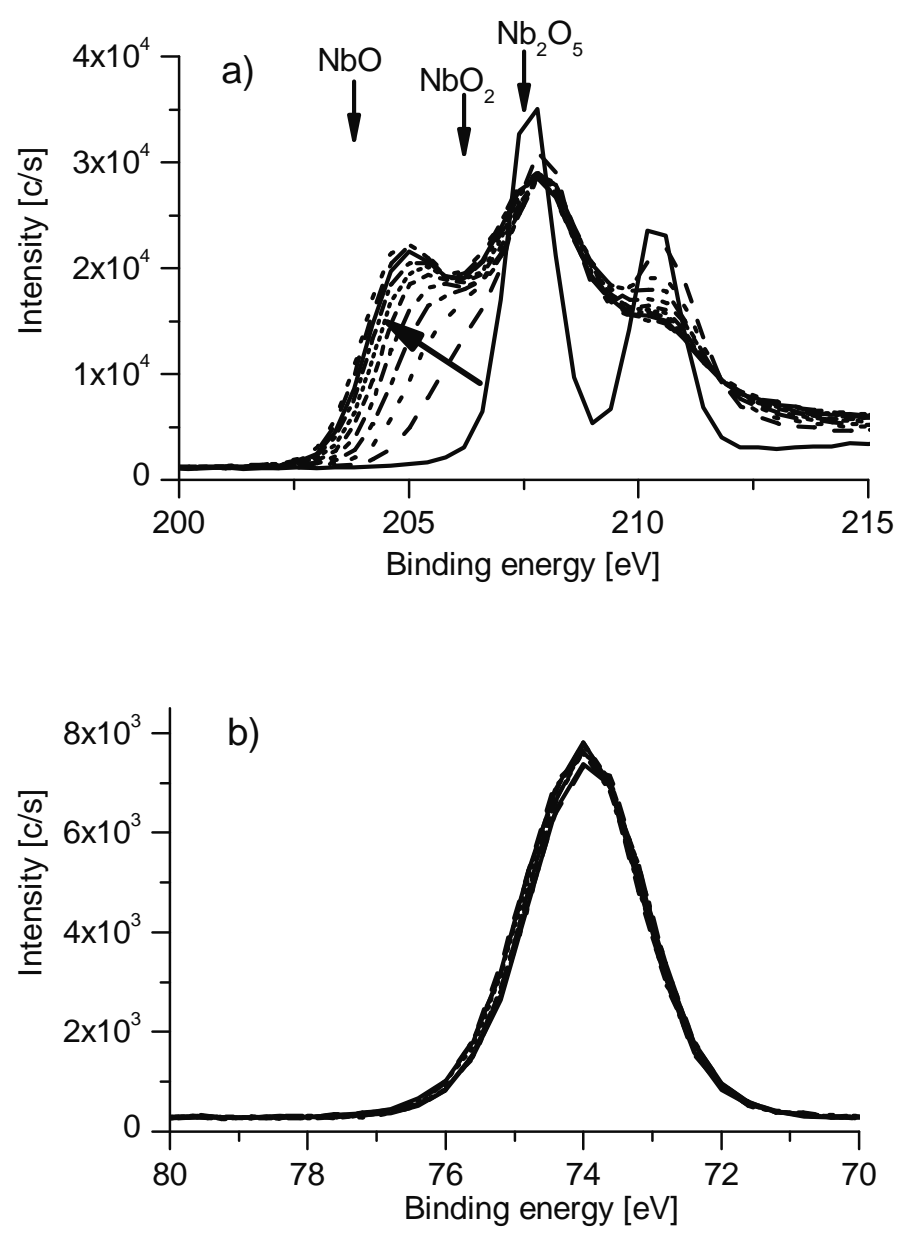

Figure 1

Page 12 of 18 

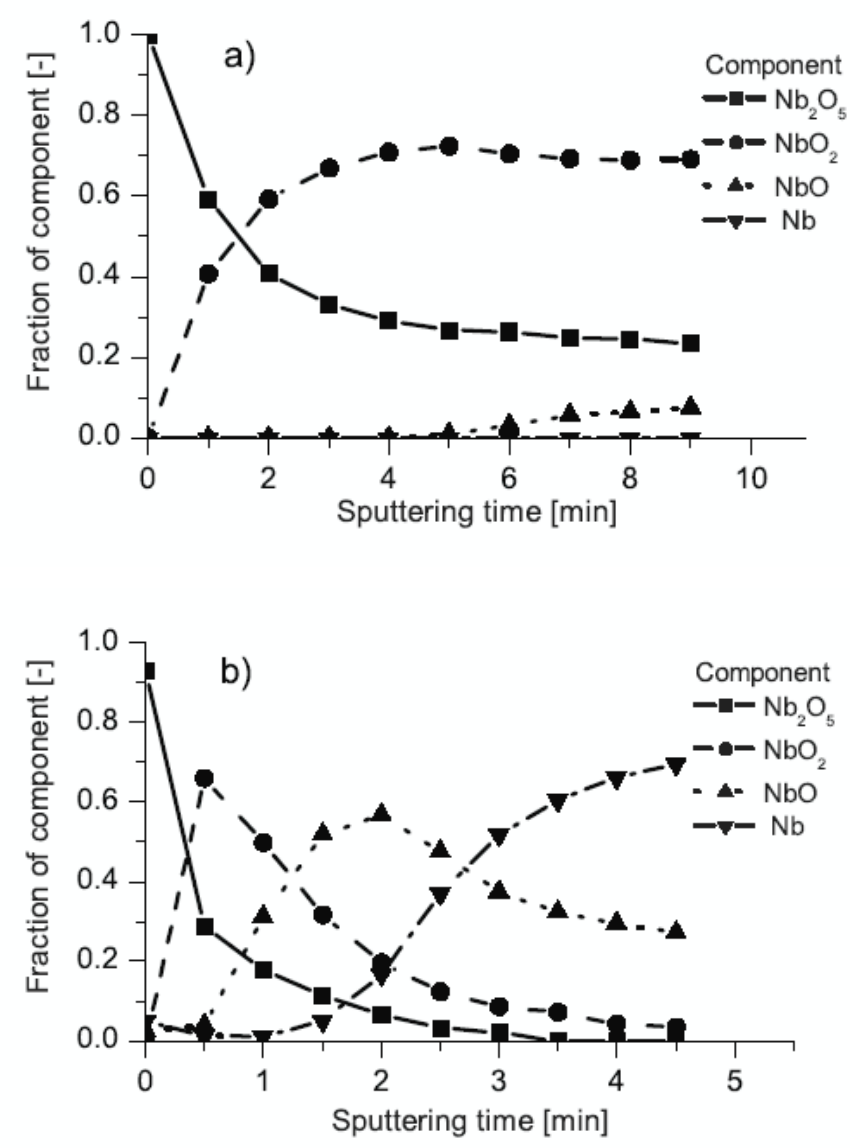

Figure 2

Page 13 of 18 

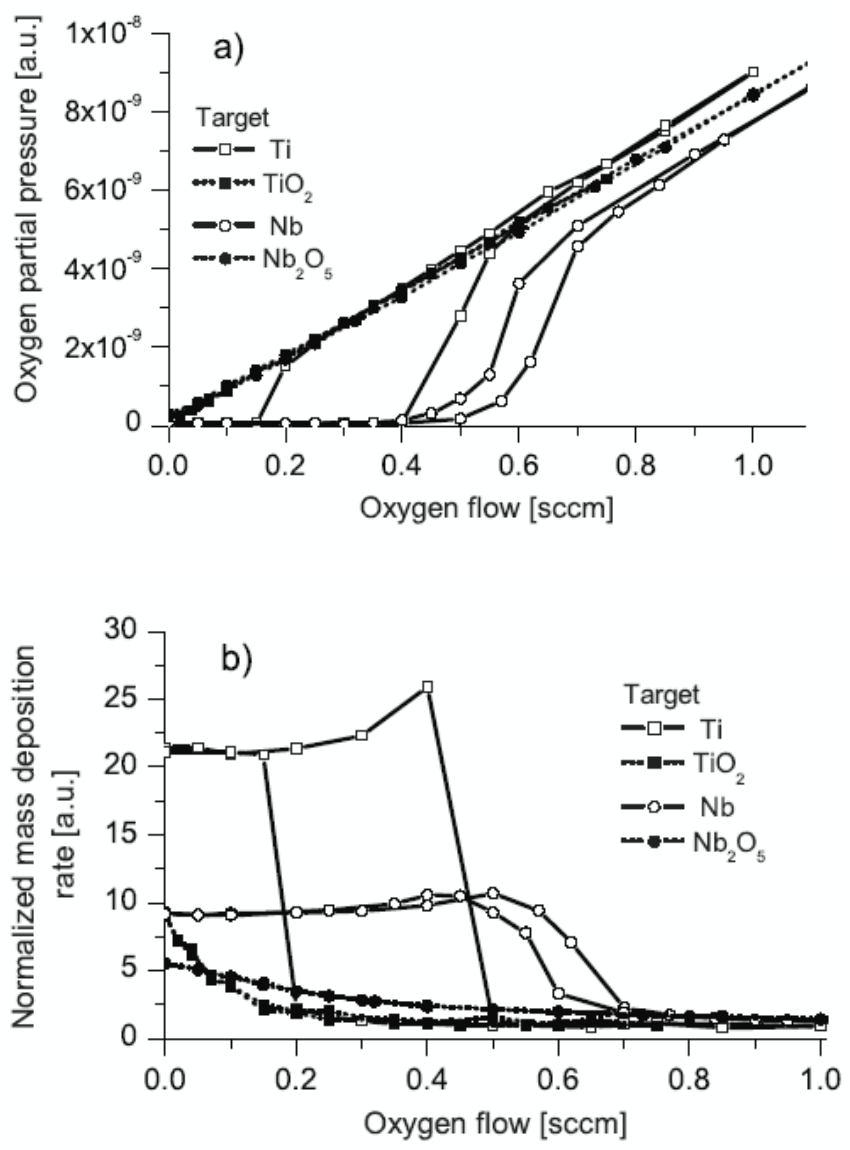

Figure 3

Page 14 of 18 


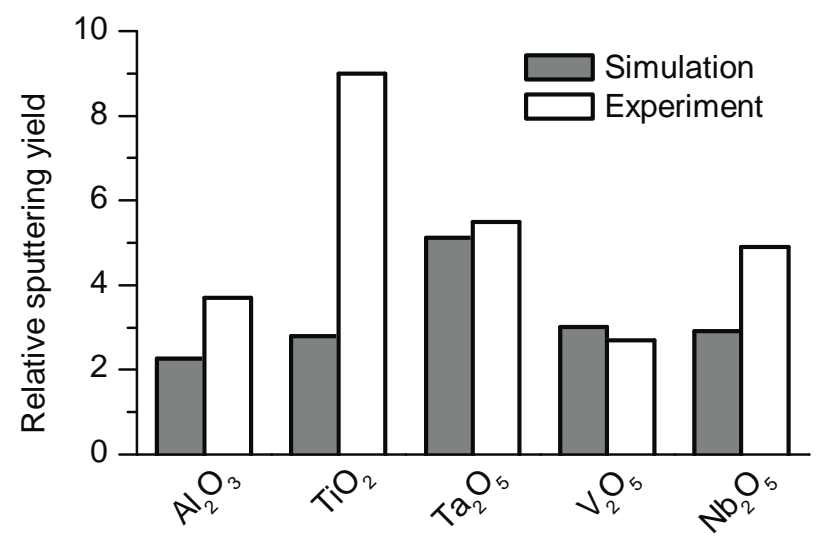

Figure 4

Page 15 of 18 


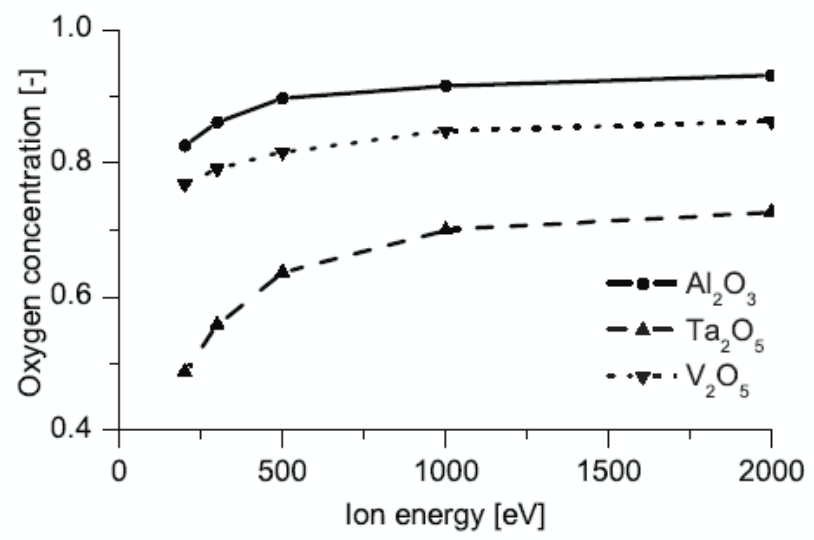

Figure 5

Page 16 of 18 


\section{References}

[1] Hofmann, S. 2000 Surf. Interface Anal. 30228

[2] Lieberman, M. A. and Lichtenberg, A. J. 2005 Principles of Plasma Discharges and Materials Processing 2nd ed. (New Jersey: Willey-Interscience).

[3] Behrisch, R. and Eckstein, W. 2007 Sputtering by Particle Bombardment 1st ed. (Heidelberg: Springer).

[4] Depla, D. and De Gryse, R. 2004 Surface \& Coatings Technology 183184

[5] Sigmund, P. 1981 Sputtering by Ion Bombardment: Theretical Concepts (Heidelberg: Springer-Verlag)

[6] Kelly, Roger 1986 Nucl. Ins. Meth. B. 18388

[7] Malherbe, J. B., Hofmann, S., and Sanz, J. M. 1986 Applied Surface Science 27355

[8] Berg, S. and Nyberg, T. 2005 Thin Solid Films 476215

[9] Sproul, W. D., Christie, D. J., and Carter, D. C. 2005 Thin Solid Films 4911

[10] Depla, D., Heirwegh, S., Mahieu, S., Haemers, J., and De Gryse, R. 2007 J. Appl. Phys. 101 013301

[11] Sproul, W. D., Graham, M. E., Wong, M. S., and Rudnik, P. J. 1997 Surface \& Coatings Technology 8910

[12] Kubart, T., Depla, D., Martin, D. M., Nyberg, T., and Berg, S. 2008 Applied Physics Letters 92221501

[13] Chang, S. J., Lee, W. C., Hwang, J., Hong, M., and Kwo, J. 2008 Thin Solid Films 516948

[14] Taglauer, E. and Heiland, W. 1978 Appl. Phys. Lett. 33950

[15] Hufner S. 2003 Photoelectron Spectroscopy 3rd ed. (Berlin: Springer).

[16] Moller, W., Eckstein, W., and Biersack, J. P. 1988 Computer Physics Communications 51355

[17] Moller, W. and Guttler, D. 2007 J. Appl. Phys. 102

[18] Moller, W. and Posselt, M. 1-4-2001 TRIDYN_FZR User Manual FZR-317) (Dresden: Forschungszentrum Rossendorf).

[19] Eckstein, W. 1991 Computer Simulation of Ion-Solid Interaction 1st ed. (Berling: SpringerVerlag). 
[20] Eckstein, W. and Biersack, J. P. 1985 Appl. Phys. A 3795

[21] Mathieu, H. J., Datta, M., and Landolt, D. 1985 J. Vac. Sci. Technol. A 3331

[22] Ohsaki, H., Tachibana, Y., Mitsui, A., Kamiyama, T., and Hayashi, Y. 2001 Thin Solid Films 392169

[23] Baretzky, B., Moller, W., and Taglauer, E. 1992 Vacuum 431207

[24] Sulyok, A., Menyhard, M., and Malherbe, J. B. 2007 Surface Science 6011857 